\title{
Le traitement des effluents de Fukushima
}

\author{
G. PAGIS ${ }^{1}$
}

(Manuscrit reçu le 14 mars 2012, accepté le 26 mai 2012)

RÉSUMÉ Après le passage du tsunami les sous-sols des bâtiments de la centrale étaient envahis par de l'eau de mer plus ou moins contaminée. L'eau d'arrosage des réacteurs accidentés s'y ajoutant, l'opérateur de la centrale s'est trouvé face à la menace d'un débordement général à brève échéance. Les groupes français AREVA et Veolia ainsi que la société américaine Kurion sont intervenus pour réaliser sur site et dans l'urgence une installation de décontamination de grande capacité. Kurion a fourni le premier étage du traitement, par échangeurs minéraux en cartouches blindées. AREVA et Veolia ont associé leurs compétences pour mettre en œuvre le procédé chimique de décontamination de Marcoule et La Hague dans des machines Veolia prévues pour le traitement d'effluents non radioactifs. Malgré l'ampleur des innovations nécessaires, et les conditions de travail sur site difficiles et changeantes, le traitement des effluents est devenu opérationnel en moins de trois mois, et a permis d'éviter le débordement.

ABSTRACT Fukushima water decontamination.

After the tsunami most of the buildings were flooded by more or less contaminated seawater. Due to the addition of water injected to cool the damaged reactors, the operator was facing the threat of a global overflow. The French companies AREVA and Veolia and American company Kurion were contracted to construct urgently a large-capacity decontamination facility. The system provided by Kurion uses mineral sorbents in shielded towers and is the first stage of decontamination. The system provided by AREVA and Veolia uses chemical precipitation as in the French nuclear plants in Marcoule and La Hague, and is implemented in Veolia compact water treatment machines, which were initially not designed for radioactive effluents. Despite the huge challenge of developing innovative systems and then building them in a difficult and changing environment, both systems were completed in less than three months, and the overflow was avoided.

Keywords: Fukushima / water / decontamination

À la fin du mois de mars 2011, l'opérateur de la centrale de Fukushima Daiichi demande l'aide d'AREVA pour gérer les effluents radioactifs qui s'accumulent sur le site.

D'un volume de l'ordre de $100000 \mathrm{~m}^{3}$, ces effluents résultent d'une part de l'intrusion d'eau de mer dans les sous-sols lors du passage du tsunami, et d'autre part de l'arrosage des cœurs des réacteurs accidentés pour assurer leur

\footnotetext{
1 AREVA EP - SGN, Département Expertise et Innovation, 25 rue de Tourville, 50120 Équeurdreville, France.
} 
refroidissement. Cet arrosage, réalisé avec de l'eau de mer les premiers jours puis avec de l'eau douce, est appelé à se poursuivre pendant plusieurs mois. Les estimations réalisées indiquent que l'ensemble du site arrivera à saturation vers le 20 juin et que si rien n'est fait les effluents radioactifs déborderont alors dans l'océan Pacifique.

L'objectif est donc de réaliser en moins de trois mois une installation de décontamination d'effluents sur le site de la centrale accidentée.

La capacité de traitement visée est de $50 \mathrm{~m}^{3} / \mathrm{h}$, pour permettre de dénoyer progressivement les bâtiments tout en poursuivant l'arrosage des cœurs avec une partie du flux d'eau décontaminée.

L'activité initiale de l'effluent varie entre $10^{5}$ et $10^{7} \mathrm{~Bq} / \mathrm{cm}^{3}$, et est principalement due au césium, au strontium et à l'iode. La décroissance radioactive de l'iode est très rapide et il n'est pas nécessaire de décontaminer l'effluent en cet élément. Le césium est la cible principale du traitement et un facteur de décontamination de l'ordre de 10000 est visé. Pour le strontium un facteur de décontamination plus faible, de l'ordre de 10, est suffisant.

Pour atteindre cet objectif AREVA s'associe à Veolia Water et propose un traitement chimique. Dans le même temps la société américaine Kurion propose un procédé de capture par échangeurs minéraux. Les deux projets progressent en parallèle et les deux systèmes seront finalement mis en œuvre en même temps, les effluents passant d'abord par le système Kurion puis par le système AREVA/Veolia.

Le système développé par Kurion utilise des zéolites optimisées pour la capture du césium, et bénéficiant du retour d'expérience de l'accident de Three Mile Island. Ces zéolites sont disposées dans des conteneurs blindés dans lesquels la circulation de l'effluent est forcée par des pompes. Quatre lignes de six conteneurs sont mises en place pour assurer à la fois la capacité et le facteur de décontamination visés (Fig. 1).

Lors du remplacement des conteneurs saturés, les opérations de déconnexion et reconnexion sont réalisées manuellement, au contact. Pour limiter les doses intégrées par le personnel d'exploitation, le blindage est très lourd, pratiquement à la limite de ce que peut supporter la structure du bâtiment d'accueil. Les remplacements de conteneurs sont déclenchés sur un seuil de débit de dose au contact.

Le système proposé par AREVA et Veolia met en œuvre des réactifs chimiques pour précipiter les éléments radioactifs sous forme de boues. Le procédé est bien 


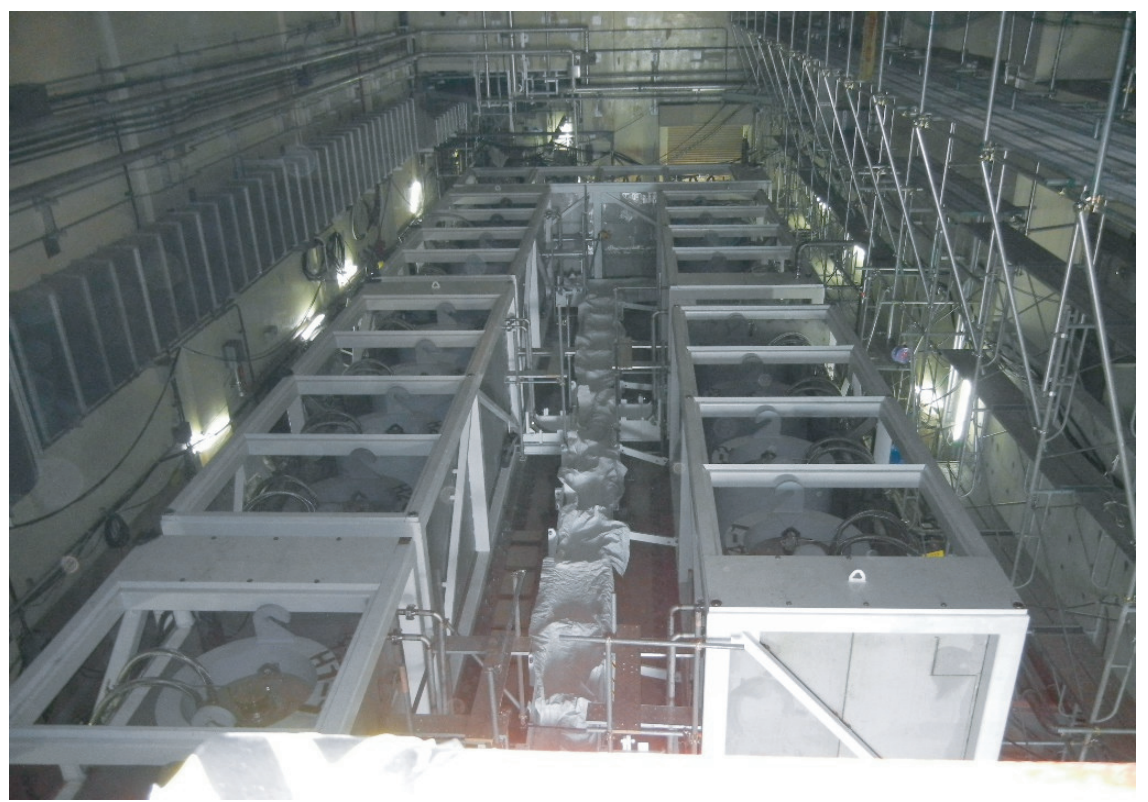

Figure 1 - Système Kurion.

Kurion system.

connu en France et est notamment mis en œuvre dans les stations de traitement des effluents de Marcoule (STEL) et La Hague (STE2 et STE3). De nombreux réactifs sont utilisés dans ces installations, mais pour les effluents de Fukushima seuls deux seront finalement retenus : le ppFeNi, précipité préformé de ferrocyanure de nickel, qui est un adsorbant du césium très efficace et très sélectif, et le sulfate de baryum qui permet l'entraînement du strontium par co-précipitation.

Les analyses réalisées par TEPCO n'ont pas révélé la présence d'autres radionucléides. Il est cependant possible que la composition des effluents soit différente au fond des bâtiments inondés. Si besoin était, le procédé pourrait être adapté sans modification d'installation, par ajout de réactifs spécifiques connus.

La mise en œuvre de ce procédé telle qu'elle est réalisée en France ne peut toutefois être envisagée pour Fukushima, la construction d'un bâtiment de type STE requérant plusieurs années. C'est le groupe Veolia qui apporte la réponse technologique. 
Veolia Water commercialise les systèmes très compacts ACTIFLO $^{\mathrm{TM}}$ et MULTIFLO $^{\mathrm{TM}}$ pour le traitement des eaux urbaines et industrielles. La précipitation des boues y est fortement accélérée par la réalisation de flocs lestés. Deux de ces systèmes se trouvant au Japon peuvent être réquisitionnés.

Les essais réalisés dans les laboratoires de Veolia, du CEA et d'AREVA permettent de conclure début avril à la compatibilité des réactifs «nucléaires » avec le procédé Veolia, et à la compatibilité de l'ensemble avec les sels marins et le bore présents dans les effluents de Fukushima.

Le facteur de décontamination obtenu en laboratoire pour le césium est de l'ordre de 100, ce qui impose la mise en œuvre de deux étages de traitement, soit deux machines Veolia.

Ces machines ne sont pas prévues pour fonctionner en environnement radioactif et de nombreuses modifications sont étudiées par AREVA : déport des pompes et vannes dans la mesure du possible, remplacement des matières plastiques, ... Les équipements sont modifiés par Veolia dans ses ateliers, puis livrés sur le site mi-mai.

Par ailleurs, les analyses de sûreté imposent la mise en place de divers systèmes pour le balayage de l'hydrogène de radiolyse, le refroidissement des boues produites, les vidanges et les rinçages. L'installation est opérée depuis une salle de conduite déportée et des protections biologiques sont installées sur les points chauds proches des zones où de la maintenance curative au contact pourrait être nécessaire. Enfin, Canberra développe un système permettant des mesures en ligne de l'activité de l'effluent, avec son spectre, ainsi que des mesures d'ambiance.

Le système Veolia ainsi « nucléarisé » est baptisé Actiflo-Rad. C'est la société japonaise JGC qui en assure la construction sur site. L'installation est réalisée dans une grande salle du bâtiment RW (Rad Waste building) (Fig. 2). L'unité de déshuilage était prévue pour le cas où l'Actiflo-Rad opèrerait seul, et n'a pas été mise en service : le déshuilage est réalisé par une autre unité similaire en amont du système Kurion. Viennent ensuite les deux machines principales Multiflo (un peu surdimensionné) et Actiflo. La clarification est très efficacement réalisée dans l'Actiflo et le filtre rotatif implanté en aval par prudence est peu sollicité. Les utilités et la distribution des réactifs sont implantées à l'extérieur.

Les boues produites sont provisoirement entreposées dans une cellule du bâtiment RW modifiée pour assurer l'agitation, le balayage, le refroidissement et la reprise. Elles seront plus tard entreposées quelques années dans des cuves ad hoc. Plusieurs procédés sont étudiés pour le conditionnement final de ces boues, à ce jour le mieux adapté semble être la cimentation. 


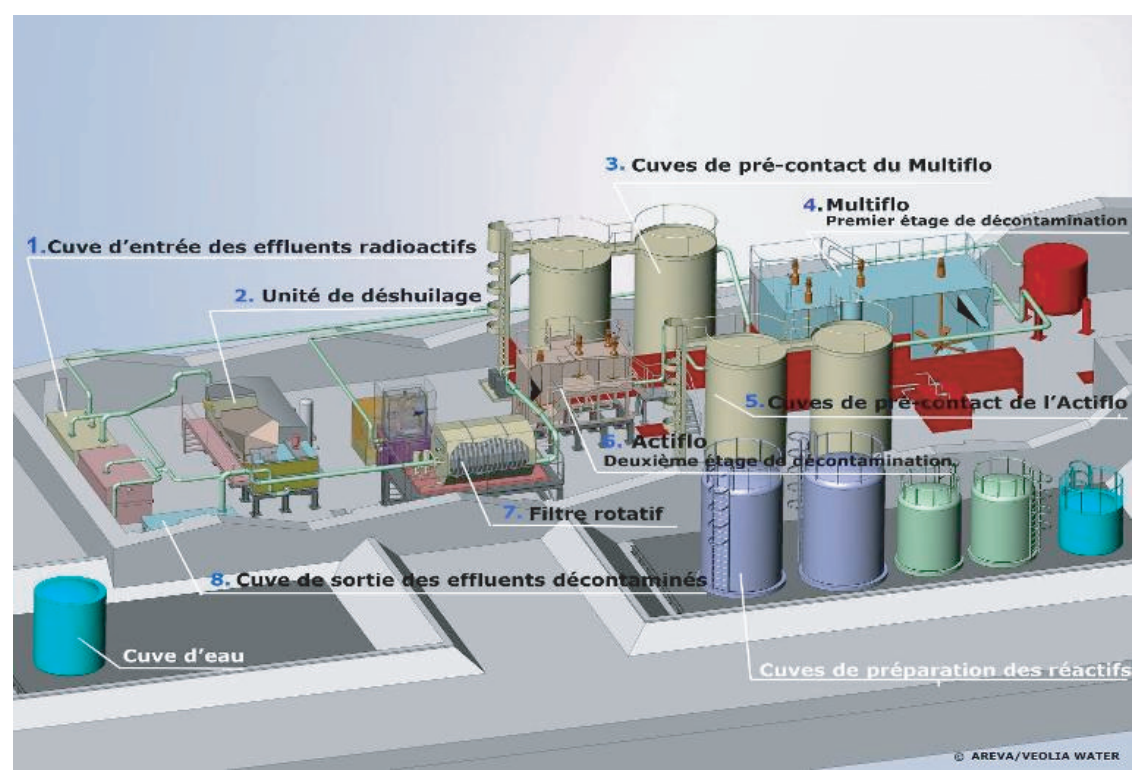

Figure 2 - Vue d'ensemble du système Actiflo-Rad. Actiflo-Rad overview.

Les essais et l'exploitation de l'Actiflo-Rad sont à charge de la société japonaise ATOX, avec une assistance AREVA/Veolia.

Les conditions de travail sur site ont évidemment considérablement compliqué les tâches de construction. Le port du masque est obligatoire sur toute la zone, ce qui limite fortement la durée de travail effectif.

Les conditions radiologiques ont évolué significativement pendant les travaux. Lors des repérages réalisés en mai par les équipes de radioprotection et d'essais d'AREVA, des débits de dose significatifs sont mesurés à l'extérieur, tant sur le trajet que sur la zone de construction. Par contre la salle du bâtiment RW dans laquelle est implanté l'essentiel du procédé est relativement protégée (Fig. 3). Les dispositions prises pour limiter l'exposition du personnel d'essais ont alors porté principalement sur la réduction du temps de transport et la suppression des escales sur site pour prendre puis rendre le dosimètre.

Par la suite il a été observé une baisse significative du débit de dose à l'extérieur du bâtiment, attribuable à la pose de plaques métalliques atténuant les radiations émises par le sol contaminé. 


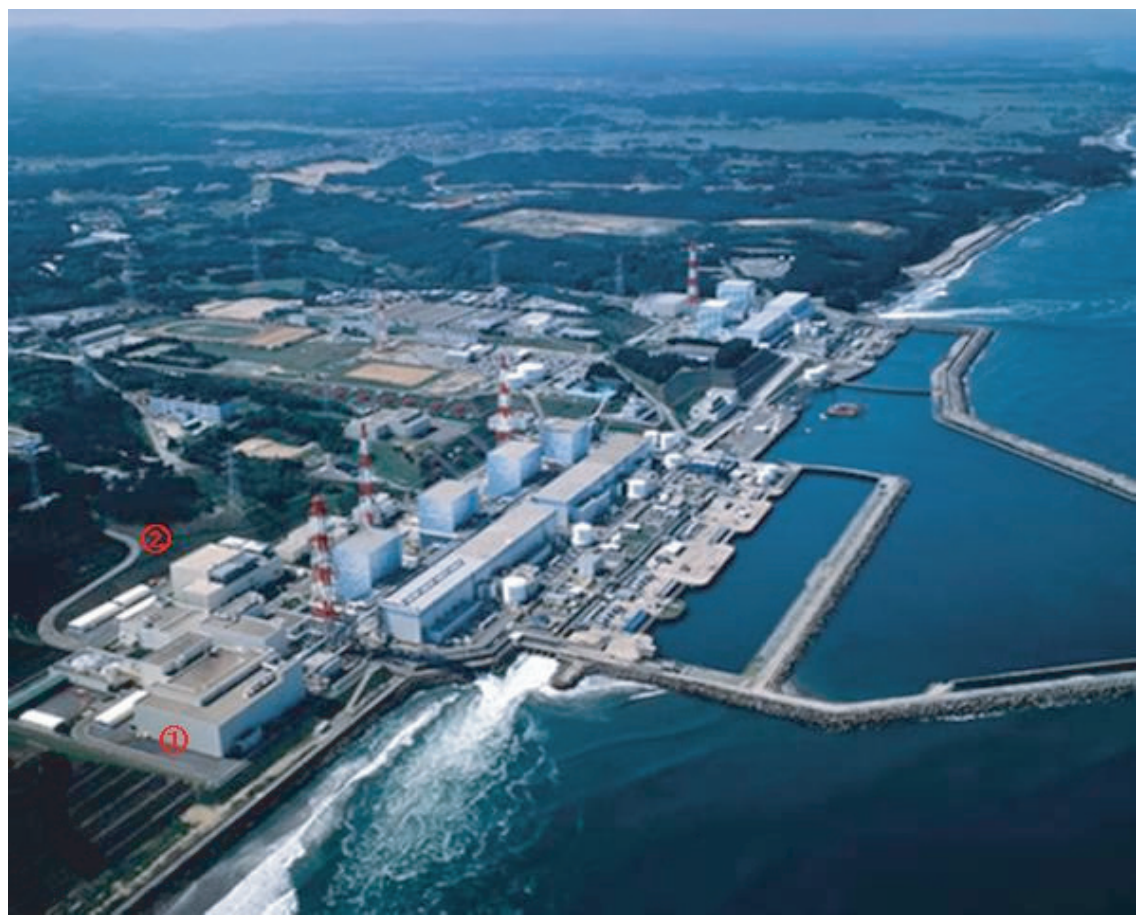

Figure 3 - Vue du site (photo prise avant l'accident). (1) Zone de construction au sud du RW building, relativement protégée. (2) Route d'accès, exposée.

View of the plant (taken before the accident). (1) Construction zone south of $R W$ building, quite protected. (2) Access road, exposed.

Par contre, dans la salle du procédé, l'ambiance s'est progressivement dégradée. La cause principale en était que les étages inférieurs du bâtiment RW participaient à l'entreposage des effluents du site, et que le niveau montait régulièrement. Quelques jours avant la mise en service actif de l'installation de décontamination certaines zones du chantier n'étaient déjà plus accessibles.

Malgré ces difficultés considérables, aggravées par la pénurie de certaines catégories de personnel de construction, l'installation de traitement des effluents a démarré à la date prévue. De nombreuses parties de l'Actiflo-Rad n'étaient cependant pas tout à fait prêtes, et le traitement a du être interrompu assez souvent pour des interventions de quelques heures. Le système Kurion, souffrant des mêmes ennuis pour les mêmes raisons, a également provoqué de nombreux arrêts. 


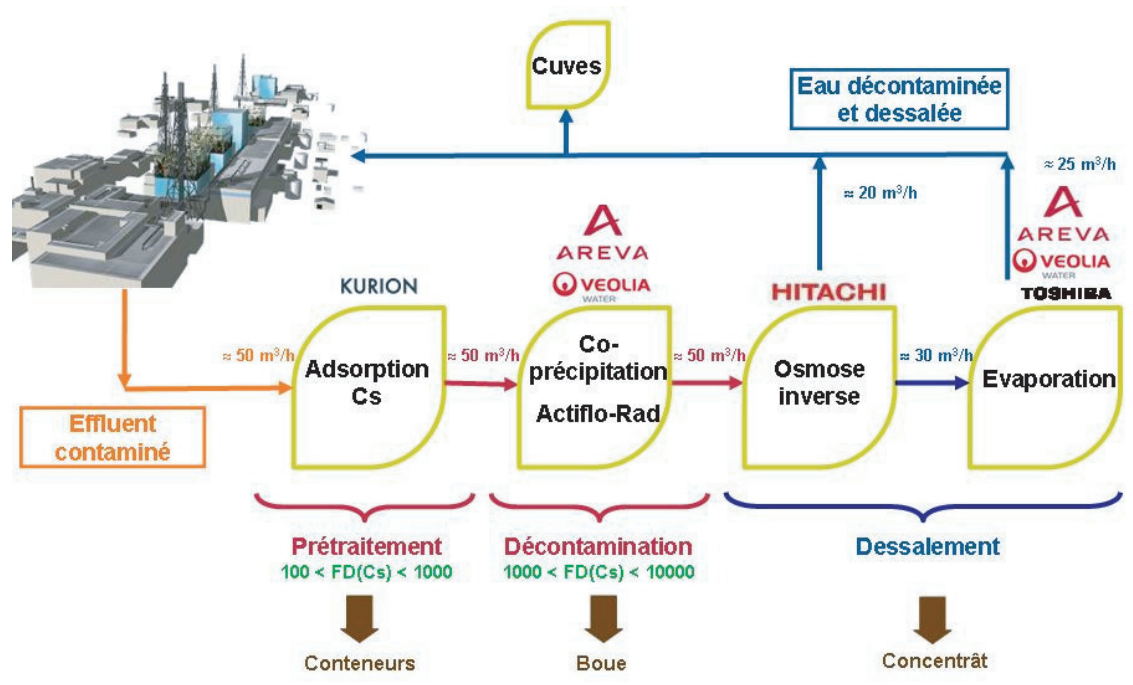

Figure 4 - Schéma général du dispositif de traitement des effluents. La décontamination (adsorption et coprécipitation) a démarré en juin, le dessalement (osmose inverse et évaporation) en août.

General diagram of the water treatment. Decontamination (adsorption and coprecipitation) started in June, desalination (reverse osmosis and evaporation) started in August.

Toutefois, bien que la qualité de finition soit inférieure à ce qu'on cherche habituellement dans l'industrie nucléaire, la disponibilité de l'installation s'est révélée suffisante et a permis de traiter les volumes souhaités : en trois mois $77000 \mathrm{~m}^{3}$ ont été décontaminés. La qualité du traitement est très bonne, avec un facteur de décontamination Cs moyen constaté de 400000 pour l'ensemble du traitement (Kurion + AREVA/Veolia).

Le dispositif de gestion des effluents de la centrale a été complété fin août 2011 par une installation de dessalement par osmose inverse et évaporation (Fig. 4).

Par ailleurs, un autre système de décontamination des effluents utilisant des échangeurs minéraux a été réalisé par TOSHIBA. Bénéficiant du REX des premières installations et d'un planning moins contraignant, ce système dénommé SARRY est plus facile à exploiter et est maintenant préférentiellement utilisé par TEPCO. 